\title{
EVIDENCE FOR STELLAR EVOLUTION IN MIRA VARIABLES
}

\author{
PATRICIA A WHITELOCK \\ South African Astronomical Observatory, $P$ O Box 9, Observatory, 7935, South Africa \\ paw@saao.ac.za
}

After briefly reviewing our understanding of Miras and their evolutionary status, three aspects of real-time evolution in these and related stars are examined:

1. Chemical changes (O-rich to $\mathrm{C}-$-rich) due to third dredge-up,

2. Period changes due to the effects of the helium shell-flash,

3. The existence of 'fossil' dust and gas shells.

Studies of resolved gas and dust shells are highlighted as particularly interesting. They will enable us to examine the mass-loss histories of many late-type stars over the last ten thousand years or so. Such observations have only recently become technically feasible and they are expecied to provide important new insight into the late stages of stellar evolution. 